\title{
Clinical characteristics, risk factors, immune status and prognosis of secondary infection of sepsis: a retrospective observational study
}

Yao Chen ${ }^{1+}$, Yanyan $\mathrm{Hu}^{1+}$, Jin Zhang ${ }^{1+}$, Yue Shen ${ }^{2}$, Junling Huang ${ }^{1}$, Jun Yin ${ }^{1}$, Ping Wang ${ }^{1}$, Ying Fan ${ }^{1}$, Jianli Wang ${ }^{1}$, Su Lu', Yilin Yang ${ }^{1}$, Lei Yan ${ }^{1}$, Keyong Li $i^{3}$ Zhenju Song ${ }^{1 *}$ (D) Chaoyang Tong ${ }^{1 *}$ and Shilin Du ${ }^{1 *}$

\begin{abstract}
Background: Secondary infection has a higher incidence in septic patients and affects clinical outcomes. This study aims to investigate the clinical characteristics, risk factors, immune status and prognosis of secondary infection of sepsis.

Methods: A four-year retrospective study was carried out in Zhongshan Hospital, Fudan University, enrolling septic patients admitted between January, 2014 and January, 2018. Clinical data were acquired from medical records. CD14 ${ }^{+}$monocyte human leukocyte antigen-D related (HLA-DR) expression and serum cytokines levels were measured by flow cytometry and enzyme-linked immunosorbent assay (ELISA) respectively.

Results: A total of 297 septic patients were enrolled, 92 of whom developed 150 cases of secondary infections. Respiratory tract was the most common site of secondary infection $(n=84,56 \%)$ and Acinetobacter baumanii the most commonly isolated pathogen $(n=40,31 \%)$. Urinary and deep venous catheterization increased the risk of secondary infection. Lower HLA-DR expression and elevated IL-10 level were found in secondary infection group. The expected prolonged in-hospital stay owing to secondary infection was $4.63 \pm 1.87$ days. Secondary infection was also associated with higher in-hospital, 30-day and 90-day mortality. Kaplan-Meier survival analysis and Log-rank test revealed that secondary infection group had worse survival between day 15 and day 90 .

Conclusions: Urinary and deep venous catheterization increased the risk of secondary infection, in which underlying immunosuppression might also play a role. Secondary infection affected the prognosis of septic patients and prolonged in-hospital length of stay.
\end{abstract}

Keywords: Sepsis, Secondary infection, Immunosuppression, HLA-DR, Cytokine

\section{Background}

Sepsis accounts for a considerable number of hospital and intensive care unit (ICU) admission and adds to the overall in-hospital mortality [1,2]. Lack of consensus and knowledge in its pathological mechanism has made the patient management difficult. With proper treatment, conditions of many septic patients became stable.

\footnotetext{
* Correspondence: song.zhenju@zs-hospital.sh.cn; tong.chaoyang@zshospital.sh.cn; du.shilin@zs-hospital.sh.cn

${ }^{\dagger}$ Yao Chen, Yanyan Hu and Jin Zhang contributed equally to this work. 'Department of Emergency Medicine, Zhongshan Hospital, Fudan University, Shanghai 200032, China

Full list of author information is available at the end of the article
}

However, some other patients developed secondary infection which led to the aggravation of disease and even the multiple organ dysfunction syndrome (MODS).

Previous studies have provided some findings on the risk factors of developing secondary infection, such as age, severity of primary disease, length of stay (LOS) in ICU and invasive procedures [3, 4]. Some studies also focused on the association between secondary infection and the prognosis of septic patients but the results were inconsistent in how secondary infection influenced the prognosis and whether it was the major cause of death $[5,6]$.

(c) The Author(s). 2019 Open Access This article is distributed under the terms of the Creative Commons Attribution 4.0 International License (http://creativecommons.org/licenses/by/4.0/), which permits unrestricted use, distribution, and 
It has also been widely studied that the underlying immune dysfunction of sepsis could lead to secondary infection. The early phase of sepsis features activated inflammation process caused by systemic release of proinflammatory cytokines called "cytokine storm" [7, 8]. Immunosuppression is then observed at later phase of sepsis as a result of the imbalance in pro- and antiinflammatory activities [9]. Sepsis could lead to a variety of mechanisms such as the apoptosis and autophagy of immune cells, endotoxin tolerance and relevant center nervous system regulation, which presented as immunosuppression consequently $[8,10,11]$. CD14 $4^{+}$monocyte human leukocyte antigen-D related (HLA-DR) expression is an effective biomarker of immune status, which reflects the comprehensive effect of pro- and antiinflammatory processes during sepsis [12-14]. Low HLA-DR expression is associated with immunosuppression and higher risk of secondary infection, especially during early phase of sepsis [15-19]. Serum cytokines levels are also commonly used by clinicians to monitor immune status. A higher release of anti-inflammatory cytokines such as IL-10, together with acute proinflammatory activities were found in the patients prone to secondary infection [20-24].

Because of the illuminating but inconsistent findings of previous studies, clinical characteristics, risk factors and the prognosis of secondary infection of sepsis were further investigated. Additionally, the association between immune status and secondary infection of sepsis based on data of HLA-DR expression and serum cytokines levels were also explored in the current study.

\section{Materials and methods}

\section{Study setting and population}

A retrospective study was carried out in emergency intensive care unit (EICU) of Zhongshan Hospital, Fudan University, Shanghai, China. Patients diagnosed with sepsis on admission between January, 2014 and January, 2018 were enrolled in this study. The diagnosis of sepsis referred to The Third International Consensus Definitions for Sepsis and Septic Shock (Sepsis-3), namely suspected infection with Sequential Organ Failure Assessment (SOFA) score $\geq 2$ [2]. Information of infection and SOFA score were acquired from Electronic Medical Record System (EMRS). Patients were excluded if they had one of the following conditions: (1) under the age of 18; (2) suffering chronic heart failure (New York Heart Function Assessment - IV), advanced malignancy, endstage liver (Child-Pugh C) or kidney diseases (CKD-5); (3) having received in-hospital treatment in other hospitals prior to admission; (4) in-hospital LOS less than 48 h. Anti-infection treatments of included patients were applied by experienced physicians based on either etiological evidence or empirical therapy plan. The study was approved by the Ethics Committee Study Board of Zhongshan Hospital, Fudan University (record number: 2006-23).

\section{Diagnosis of secondary infection}

Secondary infection was diagnosed according to $\mathrm{CDC} /$ NHSN Surveillance Definition Of Health Care-Associated Infection And Criteria For Specific Types Of Infections In The Acute Care Setting [25]. Clinical information used to identify secondary infection such as signs/symptoms and results of laboratory tests such as pathogen cultures were acquired from EMRS. Only the newly-onset nosocomial infections identified later than $48 \mathrm{~h}$ after admission were classified as secondary infections. The time of the onset of secondary infection was the day when positive cultures were collected, or when signs/symptoms emerged if no positive cultures were gained. Infections identified after leaving hospital were not documented. An infection caused by multiple pathogens but identified at the same time and same site was considered as one infection. Three experienced researchers were responsible for the diagnosis of secondary infection.

\section{Data collection}

EMRS and Computerized Physician Order Entry (CPOE) were screened for available data. The following data of each patient were collected: (1) baseline characteristics: age, gender, comorbidity and smoking history; (2) site of primary infection; (3) index of severity of the disease on admission: Acute Physiology and Chronic Health Evaluation II (APACHE II) score, SOFA score and hemodynamic status; (4) interventions such as the use of glucocorticoids, anticoagulation therapy, mechanical ventilation, urinary catheterization, deep venous catheterization, continuous renal replacement therapy and blood transfusion (whether those interventions were applied before or after the onset of secondary infection was noticed); (5) occurrence time, site and pathogen of secondary infection; (6) LOS in hospital and ICU, the outcome of hospital stay.

\section{Measurement of monocyte HLA-DR expression and serum levels of cytokines}

In order to explore the underlying immune mechanism of secondary infection, we acquired the data from Database of Clinical Sample and Information for Sepsis of Zhongshan Hospital, an database founded in 2008 and intended for the collection and perseveration of clinical samples of septic patients. According to the guideline of database, the peripheral blood samples were collected in the BD Vacutainer tubes (BD Biosciences, CA, USA) at day 1, 3 and 7 after admission. In some patients, samples at day 3 and 7 were not collected due to specific clinical conditions. Thus, data of only a part of the included patients were available as the limitation of a retrospective 
study. To explore CD14 ${ }^{+}$HLA-DR $^{+}$monocytes expression, a following double color staining was utilized: a fluorescein conjugated (FITC)-CD14, allophycocyanin conjugated (APC)-HLA-DR (BD Biosciences, CA, USA), according to manufacturer's instructions. Appropriate isotype controls were run with healthy controls and used for compensation and gating blood samples. Subsequently, samples were analyzed on a 18-parameter $\mathrm{BD}$ LSR Fortessa analyzer (BD Biosciences, CA, USA) with FlowJo software (Tree Star Inc., OR, USA). HLA-DR expression was shown as the percentage of $\mathrm{CD} 14^{+}$HLA$\mathrm{DR}^{+}$monocytes among all $\mathrm{CD} 14^{+}$monocytes. The levels of tumor necrosis factor- $\alpha$ (TNF- $\alpha$ ), interleukin-6 (IL-6), IL- 8 and IL-10 were measured by ELISA method (R\&D System, MN, USA) according to manufacturer's instructions. The experiments of flow cytometry and ELISA were conducted right after the samples were collected and the results were recorded in the database. In this retrospective study, the results were directly acquired from the database.

\section{Statistical analysis}

The Kolmogorov-Smirnov test was used to verify the normality of all data. Normally distributed data were expressed as means and standard deviations (SD). Abnormally distributed continuous data were expressed as medians with the 25th and 75th quartiles. Categorical data were expressed as frequency and percentage.

The risk factors of secondary infection of septic patients were explored by a two-step method. Firstly, univariate analysis was conducted. Covariates included age, gender, comorbidities, smoking history, site of primary infection, hemodynamic status and severity of disease on admission, HLA-DR expression, serum cytokines levels and clinical interventions before onset of secondary infection. Student's t test was used to compare normally distributed data and Mann-Whitney $U$ test was utilized to compare abnormally distributed data. Categorical data were compared by Pearson's chi-square test or Fisher's exact test when appropriate. Secondly, covariates with statistical significance in univariate analysis were tested in multivariate binary logistic regression analysis to identify the independent risk factors by means of Backward: Conditional method. Because of the data missing of HLA-DR expression and serum cytokines levels, they were not brought into multivariate analysis. Dynamic changes of HLA-DR expression and serum cytokines levels were also statistically evaluated by comparing the levels of biomarkers between different points in time using Mann-Whitney $U$ test.

In our study, we treated in-hospital LOS as an outcome of secondary infection, rather than a potential risk factor. A multistate model with 4 states (state 0 : admission, state 1: development of secondary infection, state 2: being discharged alive, state 3: in-hospital death) was performed using "etm" package in $\mathrm{R}$ in order to explore the influence of secondary infection on in-hospital LOS $[3,26]$, where the data of patients with an in-hospital LOS longer than 100 days were omitted to eliminate the impact of extreme cases (see Additional file 1: Figure S1). Survival analysis was conducted using Kaplan-Meier method. Log-rank test was used to compare survival curves and it was conducted in every division once two curves had intersections. The two-step method was also used to explore the risk factors of mortality. Univariate analysis was conducted first and followed by multivariate binary logistic regression analysis. Secondary infection was among covariates, together with age, gender, comorbidities, smoking history, site of primary infection, hemodynamic status and severity of disease on admission, clinical interventions and in-hospital and ICU LOS.

All statistical analyses were two-sided, and the significance level was set to $P<0.05$. We checked the model assumptions before using each statistical method. Statistical analysis was conducted on SPSS 25.0 (SPSS Inc., IL, USA) and R 3.5.1 (R Development Core Team).

\section{Results}

\section{Characteristics of septic patients}

From January, 2014 to January, 2018, a total of 297 patients were enrolled. A flowchart to illustrate the recruitment process was shown in Fig. 1. Among all included patients, 195 were men and the median age was 66 years. Two hundred forty-one patients had comorbidities (81.1\%). Respiratory tract was the most common site of primary infection $(n=216,72.7 \%)$. Other sites of infection included abdomen $(n=62,20.9 \%)$, urinary tract $(n=22,7.4 \%)$, skin and soft tissue $(n=12,4 \%)$ and blood stream $(n=4,1.3 \%), 21$ patients had more than one infection sites (7.1\%). Seventy-seven patients had septic shock on admission (25.9\%). The baseline characteristics of the enrolled patients were shown in Table 1.

\section{Characteristics of septic patients with secondary infection} One hundred fifty cases of secondary infection were developed in 92 patients, 26 of whom had multiple secondary infections. Respiratory tract was the most common site of secondary infection $(n=84,56 \%)$, followed by urinary tract $(n=42,28 \%)$, blood stream and disseminated infection $(n=18,12 \%)$, abdomen $(n=5$, $3.3 \%)$ and skin and soft tissue $(n=1,0.7 \%)$. Day 8 was the median time of developing the first secondary infection. Acinetobacter baumanii $(n=40,26.7 \%)$, Klebsiella pneumoniae ( $n=21,14 \%)$, Enterococcus faecium ( $n=11$, $7.3 \%)$, Candida tropicalis $(n=9,6 \%)$, Pseudomonas aeruginosa $(n=9,6 \%)$ and Staphylococcus aureus $(n=9,6 \%)$ were common identified pathogens. In 23 cases, pathogens were not identified. The characteristics of secondary 


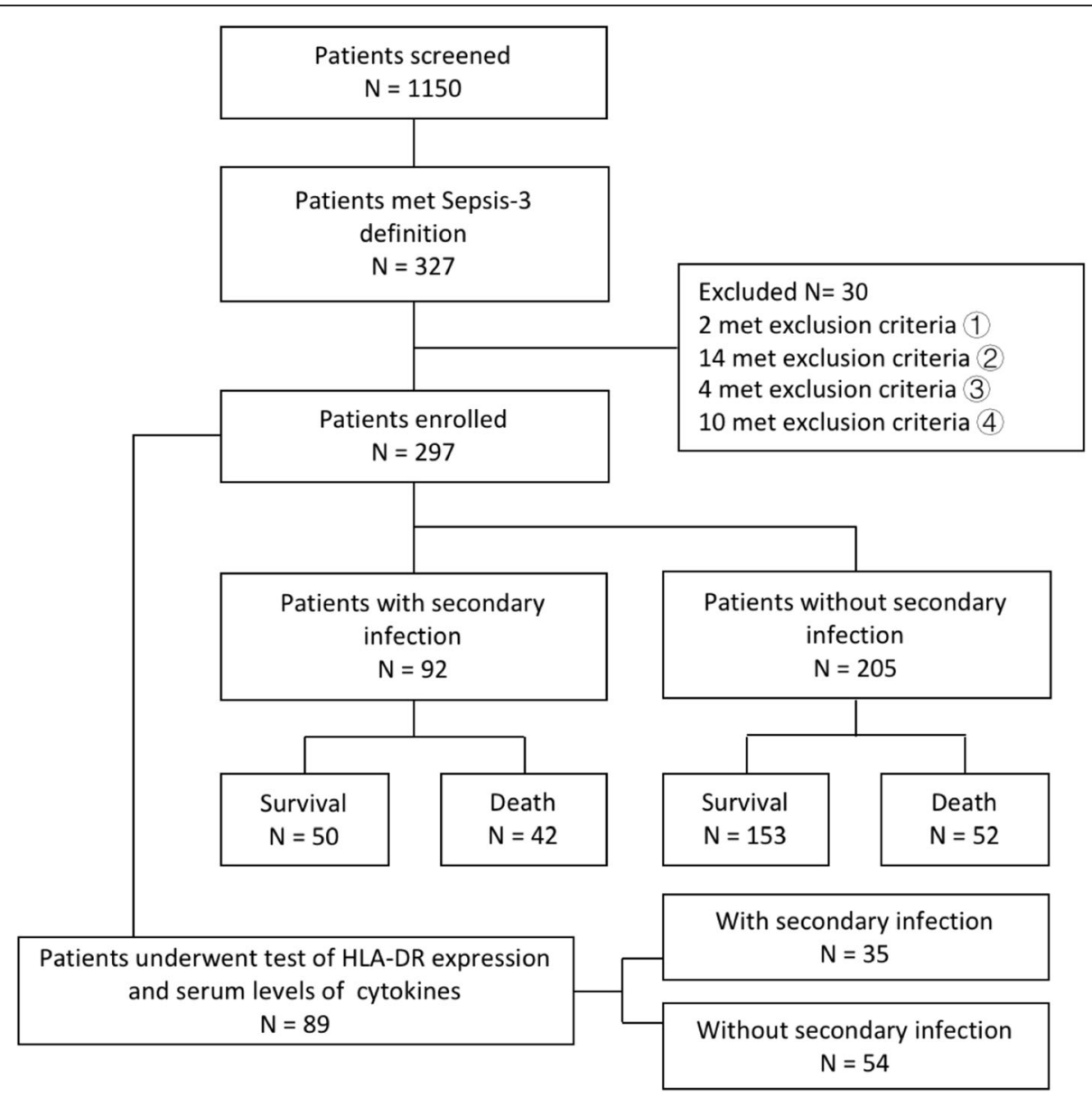

Fig. 1 Study flowchart

infections were shown in Table 2 and time of onset, distribution of pathogen and diagnostic criterion of each infection were shown in Additional file 2: Table S1.

\section{Risk factors of secondary infection in septic patients}

No statistical significance existed between septic patients with and without secondary infection concerning age, gender, comorbidity and site of primary infection. In univariate analysis, statistical significance was found in severity of illness on admission (APACHE II score: $P=$ 0.001 ; SOFA score: $P=0.007$ ) and some interventions before the onset of secondary infection such as the use of mechanical ventilation (OR 2.752, 95\% CI 1.604 to 4.721, $P<0.001$ ), urinary catheterization (OR 5.292, 95\% CI 2.997 to $9.343, P<0.001)$, deep venous catheterization (OR 4.494, 95\% CI 2.629 to 7.680, $P<0.001$ ) and blood transfusion (OR 2.152, 95\% CI 1.18 to $3.925, P=0.011$ ) (Table 1). Factors with statistical significance were tested under multivariate logistic regression analysis and urinary catheterization (OR 3.384, 95\% CI 1.791 to 6.392, $P<0.001$ ) and deep venous catheterization (OR 2.608,
95\% CI 1.422 to $4.784, P=0.002)$ remained statistical significant (Table 3).

The association between immune dysfunction and secondary infection of sepsis

Data of a part of patients were available for HLA-DR expression and cytokines. The exact numbers were shown in the legend of Table 1. In the univariate analysis of the risk factors of secondary infection, statistical significance was found in HLA-DR expression at day $3(P=0.048)$, IL-6 level at day $1(P=0.025)$, IL-8 level at day $3(P<$ $0.001)$ and IL-10 level at day $7(P=0.035)$. The results were shown in Table 1 and Fig. 2. Although statistical significance was not found at every time point, a trend of decrease of HLA-DR expression and increase of IL-10 level in secondary infection group was observed, which is indicative of immunosuppression (Fig. 2a and b). Interestingly, a reverse trend of dynamic change was found between two pro-inflammatory cytokines IL-6 and IL-8 in both secondary infection and non-secondary infection groups (Fig. 2c and d). Dynamic changes of those markers were statistically significant between certain 
Table 1 Characteristics of septic patients classified according to developing secondary infection or not

\begin{tabular}{|c|c|c|c|}
\hline & With secondary infection $n=92$ & Without secondary infection $n=205$ & $P$ value \\
\hline \multicolumn{4}{|l|}{ Baseline characteristics } \\
\hline Age, median (25th,75th) & $66.5(53.5-78.8)$ & $65(52.3-75)$ & 0.323 \\
\hline$>65$ years, $n(\%)$ & $50(54.3)$ & $105(51.2)$ & 0.618 \\
\hline Men, n (\%) & $63(68.5)$ & $132(64.4)$ & 0.493 \\
\hline \multicolumn{4}{|l|}{ Comorbidities, n (\%) } \\
\hline None & $16(17.4)$ & $40(19.5)$ & 0.666 \\
\hline Hypertension & $42(45.7)$ & $82(40)$ & 0.361 \\
\hline Other cardiovascular diseases ${ }^{a}$ & $15(16.3)$ & $25(12.2)$ & 0.337 \\
\hline Diabetes mellitus & $23(25)$ & $46(22.4)$ & 0.629 \\
\hline Cerebrovascular diseases & $6(6.5)$ & $13(6.3)$ & 0.953 \\
\hline Respiratory diseases & $9(9.8)$ & $23(11.2)$ & 0.712 \\
\hline Hepatitis and cirrhosis & $3(3.3)$ & $10(4.9)$ & 0.761 \\
\hline Renal insufficiency & $4(4.3)$ & $15(7.3)$ & 0.334 \\
\hline Malignancy & $8(8.7)$ & $17(8.3)$ & 0.908 \\
\hline Immunosuppression & $12(13)$ & $24(11.7)$ & 0.744 \\
\hline Smoker, n (\%) & $32(34.8)$ & $69(33.7)$ & 0.85 \\
\hline \multicolumn{4}{|l|}{ Site of infection, n (\%) } \\
\hline Respiratory tract & $70(76.1)$ & $146(71.2)$ & 0.384 \\
\hline Abdomen & $15(16.3)$ & $47(22.9)$ & 0.194 \\
\hline Urinary tract & $8(8.7)$ & $14(6.8)$ & 0.57 \\
\hline Skin and soft tissue & $6(6.5)$ & $6(2.9)$ & 0.203 \\
\hline Blood stream & $2(2.2)$ & $2(1)$ & 0.59 \\
\hline More than one sites & $8(8.7)$ & $13(6.3)$ & 0.464 \\
\hline In shock on admission, n (\%) & $28(30.4)$ & $49(22.4)$ & 0.235 \\
\hline \multicolumn{4}{|l|}{ Severity of disease, median (25th,75th) } \\
\hline APACHE II score & $17(9.25-22)$ & $11(7-18)$ & 0.001 \\
\hline SOFA score & $4(3-8)$ & $4(2.5-6)$ & 0.007 \\
\hline \multicolumn{4}{|l|}{ Monocyte HLA-DR expression (\%) } \\
\hline Day 1 , mean (SD) & $31.6(14.3)$ & $34.5(14.9)$ & 0.364 \\
\hline Day 3, median (25th,75th) & $28.6(18.8-42)$ & $41.1(27.5-50.4)$ & 0.048 \\
\hline Day 7 , median (25th,75th) & $29.6(14.3-35.1)$ & $33.2(13.8-65.4)$ & 0.722 \\
\hline \multicolumn{4}{|l|}{ Levels of serum cytokines $(\mathrm{pg} / \mathrm{ml})^{\mathrm{b}}$} \\
\hline \multicolumn{4}{|l|}{ Day 1 , median (25th,75th) } \\
\hline IL-6 & $26.8(13.6-363.5)$ & $21.1(7.5-58.2)$ & 0.025 \\
\hline IL-8 & $36.3(18.7-70)$ & $22.9(12-77.5)$ & 0.375 \\
\hline IL-10 & $8.6(5.4-24)$ & $10(9.3-17.8)$ & 0.121 \\
\hline \multicolumn{4}{|l|}{ Day 3, median (25th,75th) } \\
\hline IL-6 & $628.5(23.5-1694.5)$ & $640.5(17.8-942.3)$ & 0.478 \\
\hline IL-8 & $29.89(20-106)$ & $8(4.9-15.6)$ & $<0.001$ \\
\hline $\mid \mathrm{LL}-10$ & $21.7(6.4-35.3)$ & $14.6(5-27.7)$ & 0.303 \\
\hline \multicolumn{4}{|l|}{ Day 7} \\
\hline IL-6, median (25th,75th) & $921(652-1377)$ & 754 (584-1004) & 0.226 \\
\hline IL-8, median (25th,75th) & $11.1(6-41.8)$ & $12.5(5.7-14)$ & 0.79 \\
\hline IL-10, mean (SD) & $60.6(47.3)$ & $16.8(10.4)$ & 0.035 \\
\hline
\end{tabular}


Table 1 Characteristics of septic patients classified according to developing secondary infection or not (Continued)

\begin{tabular}{|c|c|c|c|}
\hline & With secondary infection $n=92$ & Without secondary infection $n=205$ & $P$ value \\
\hline \multicolumn{4}{|l|}{ Interventions, $\mathrm{n}(\%)^{c}$} \\
\hline Glucocorticoid & $46(50)$ & $80(39)$ & 0.077 \\
\hline Anticoagulation therapy & $33(35.9)$ & $66(32.2)$ & 0.535 \\
\hline Mechanical ventilation & $68(74)$ & $104(50.7)$ & $<0.001$ \\
\hline Urinary catheterization & $72(78.3)$ & $83(40.5)$ & $<0.001$ \\
\hline Deep venous catheterization & $66(71.7)$ & $74(36.1)$ & $<0.001$ \\
\hline Continuous renal replacement therapy & $11(12)$ & $19(9.3)$ & 0.477 \\
\hline Blood transfusion & $25(27.2)$ & $30(14.6)$ & 0.011 \\
\hline \multicolumn{4}{|l|}{ LOS (days), median (25th,75th) } \\
\hline In-hospital & $23.5(12-34)$ & $22(10-32.5)$ & $<0.001$ \\
\hline ICU & $11(7-17)$ & $11(6-16.5)$ & $<0.001$ \\
\hline \multicolumn{4}{|l|}{ Mortality, n (\%) } \\
\hline In-hospital & $42(45.7)$ & $52(25.4)$ & 0.001 \\
\hline 30-day & $32(34.8)$ & $48(23.4)$ & 0.041 \\
\hline 90-day & $39(42.4)$ & $52(25.4)$ & 0.003 \\
\hline
\end{tabular}

${ }^{a}$ Other cardiovascular diseases included coronary heart disease, arrhythmia, myocardiosis and valvular heart disease

${ }^{b}$ Data of 89, 77 and 21 patients were available for HLA-DR expression at day 1, 3 and 7 respectively, in which 35,34 and 12 patients developed secondary infection. And data of 87,38 and 18 patients were available for cytokines at day 1, 3 and 7 respectively, in which 33,18 and 8 patients developed secondary infection

'In the group of secondary infection, it referred to the interventions before the onset of secondary infection

points in time, the results were shown in Fig. 2 and Additional file 3: Table S2. Representative flow cytometry profiles for HLA-DR expression were shown in Fig. 3.

\section{The association between secondary infection and the outcomes of sepsis}

Secondary infection group had longer LOS in hospital and ICU than non-secondary infection group (in-hospital LOS: $P<0.001$; ICU LOS: $P<0.001$ ) (Table 1). Multistate model revealed expected prolonged LOS in hospital was 4.63 days based on a standard error of 1.87 days (Fig. 4). In-hospital, 30-day, 90-day mortality was $45.7,34.8,42.4 \%$ in secondary infection group and 25.4, 23.4 and $25.4 \%$ in non-secondary infection group respectively (OR $2.472,95 \%$ CI 1.474 to $4.145, P=0.001$; OR $1.744,95 \%$ CI 1.019 to $2.985, P=0.041$; OR 2.165, $95 \%$ CI 1.288 to $3.640, P=0.003$, respectively). The proportion of developing secondary infection were 44.7 and $24.6 \%$ in in-hospital mortality group and survival group respectively (OR $2.472,95 \% \mathrm{CI} 1.474$ to $4.145, P=0.001$ ) (see Additional file 4: Table S3). Multivariate binary logistic regression analysis also found out that secondary infection was an independent risk factor of in-hospital mortality (OR 3.476, 95\% CI 1.599 to $8.219, P=0.003$ ) (see Additional file 5: Table S4). Kaplan-Meier survival curves and Log-rank test revealed no difference between two groups before day $15(P=0.426)$ (see Additional file 6: Figure S2). But non-secondary infection group had a better survival between day 15 and day $90(P<0.001)$ (Fig. 5) and subgroup analysis showed that the difference remained significant in both groups of patients with and without septic shock $(P=0.04$ and $P<0.001)$ (see Additional file 7: Figure S3).

\section{Discussion}

Our study confirmed a high incidence of secondary infection in septic patients (31.0\%) and suggested urinary and deep venous catheterization could bring higher risk of developing secondary infection, in which immunosuppression might be the underlying mechanism. Secondary infection also affected the outcomes, which featured poor survival at later period ( $>15$ days after admission) and expected prolonged in-hospital LOS of $4.63 \pm 1.87$ days.

We found that secondary infections mostly developed in respiratory tract and were caused by Gram-negative bacteria. This finding was consistent with previous studies. A recent meta-analysis revealed that lower respiratory tract was the most common site of nosocomial infection in general hospital and Pseudomonas aeruginosa, Escherichia coli, Acinetobacter baumannii and Klebsiella pneumoniae were among most common pathogens [27]. There's a study suggesting that the high pathogenicity of such Gram-negative bacteria was due to drug resistant and invasive procedures which served as approaches of the invasion of pathogens [28]. It has been increasingly acknowledged that increased susceptibility of secondary infection could be pathogen-specific due to the different patterns of immune barrier destruction which caused opportunistic bacterial and fungal infections, as well as 
Table 2 Characteristics of secondary infections

\begin{tabular}{|c|c|}
\hline \multicolumn{2}{|l|}{ Site of infection, $\mathrm{n}(\%)^{\text {a }}$} \\
\hline \multicolumn{2}{|l|}{ Respiratory tract } \\
\hline PNU & $83(55.3)$ \\
\hline LUNG & $1(0.7)$ \\
\hline \multicolumn{2}{|l|}{ Urinary tract } \\
\hline SUTI & $41(27.3)$ \\
\hline OUTI & $1(0.7)$ \\
\hline \multicolumn{2}{|l|}{ Blood stream and disseminated infection } \\
\hline LCBI & $12(8)$ \\
\hline DI & $6(4)$ \\
\hline \multicolumn{2}{|l|}{ Abdomen } \\
\hline $\mid A B$ & $4(2.7)$ \\
\hline GIT & $1(0.7)$ \\
\hline \multicolumn{2}{|l|}{ Skin and soft tissue } \\
\hline ST & $1(0.7)$ \\
\hline \multicolumn{2}{|l|}{ Time of onset of the first identified secondary infection } \\
\hline Median (25th,75th) & $8(5.25,14)$ \\
\hline \multicolumn{2}{|l|}{ Time range, $\mathrm{n}(\%)$} \\
\hline day 3 & $5(5.4)$ \\
\hline$>$ day $3, \leq$ day 7 & $36(39)$ \\
\hline$>$ day 7, sday 15 & $33(35.9)$ \\
\hline$>$ day 15 & 18 (19.6) \\
\hline Patients with multiple secondary infections, n (\%) & $26(28.3)$ \\
\hline Secondary infection without identified pathogens, n (\%) & $23(15.3)$ \\
\hline
\end{tabular}

higher chance of viral reactivation and co-infection [29-31]. In our study, 6 cases of secondary infections were defined as disseminated infections, which were likely caused by viruses according to the CDC criteria [25]. However, it's possible that some pathogenic microorganisms, especially viruses, were not identified due to limited testing technologies.

We found higher APACHE II and SOFA scores on admission in patients with secondary infection, which were similar to previous studies $[3,4,32]$. Although illness severity was not found to be an independent risk factor of secondary infection in this study, it could be explained that the more severe patients died mostly at the very early period of disease before developing secondary infections, which might impact the true association between the risk and illness severity.

It's widely acknowledged that catheter indwelling was a major cause of nosocomial infection [33-35]. We found that urinary catheterization was an independent risk factor of secondary infection. Another study revealed that catheterassociated urinary tract infection was not only affected by duration of urinary catheterization, but also the presence of another site of nosocomial infection [36], which was confirmed by our study that many cases of secondary infections in urinary tract were subsequent to secondary infections at other sites. Deep venous catheterization was also common in ICU setting and our finding was consistent with the study by van Vught et al. that it was also an independent risk factor of secondary infection [4]. It's also proved by previous studies that the need for mechanical ventilation of critical ill patients incurred high prevalence of ventilator-associated pneumonia, which accounted for nearly half of nosocomial infections [3, 4, 37]. Blood transfusion was also a potential risk factor due to the effect of transfusion-related immune modulation (TRIM) as reveal by previous studies [38-41]. Nevertheless, mechanical ventilation and blood transfusion were only found to be risk factors in univariate analysis of our study, but not multivariate analysis. It might be explained by the lack of the discrimination of invasive and non-invasive ventilation, the length of ventilation and the quantity and type of blood transfusion due to limited medical records.

Immune status of septic patients and its underlying mechanism have been widely studied. Innate immune function was compromised due to the dysfunction of neutrophils, monocytes, dendritic cells and myeloidderived suppressor cells (MDSCs) which caused altered first-line of defense, inhibition of $\mathrm{T}$ cell proliferation, altered inflammatory response and incomplete activation of $\mathrm{T}$ cells [8]. Adaptive immune function was also compromised as sepsis affected the effector functions and phenotypes of $\mathrm{T}$ cells, B cells and innate-type lymphocytes [8]. HLA-DR and cytokines were chosen to reflect the immune status in this study. HLA-DR was a marker reflecting both innate and adaptive immune function and lower expression indicated immunosuppression [8]. IL-10 was an anti-inflammatory cytokine and elevated level reflected the down-regulation of inflammation process. It might generate MDSCs and enhance the immunosuppression during sepsis [20, 42]. In secondary

Table 3 Results of multivariate logistic regression test of the risk factors of secondary infection

\begin{tabular}{lllllll}
\hline Variables $^{\mathrm{a}}$ & Partial regression coefficient & Standard error & Wald X2 & $P$ value & OR & $95 \%$ Cl \\
\hline Urinary catheterization & 1.219 & 0.325 & 14.109 & $<0.001$ & 3.384 & $1.791-6.392$ \\
Deep venous catheterization & 0.959 & 0.309 & 9.601 & 0.002 & 2.608 & $1.422-4.784$ \\
\hline
\end{tabular}

${ }^{a}$ Analysis was conducted by method Backward: Conditional. Variable blood transfusion was removed on step 2, mechanical ventilation on step 3, APACHE II score on step 4 and SOFA score on step 5 

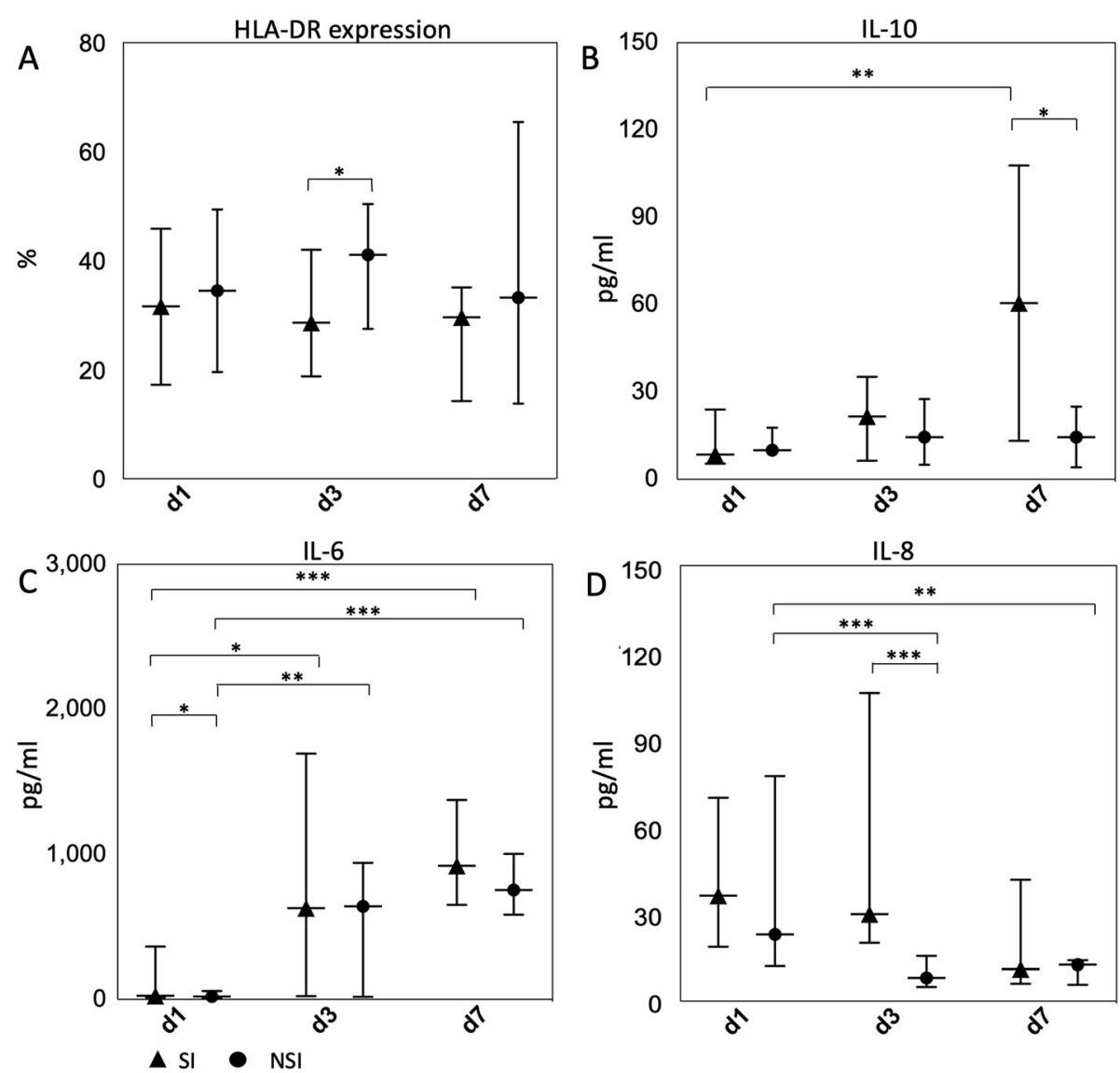

Fig. 2 Biomarkers of immune status in septic patients stratified according to developing secondary infection or not. Data of a part of patients were available for HLA-DR expression and cytokines and the exact numbers were shown in Table 1. Data were presented as medians (shown as triangles or circles) and 25- and 75- percentile error bars. Exceptions were mean and standard deviation error bars were used in HLA-DR expression at day 1 and IL-10 level at day 7. a and $\mathbf{b}$ represented the levels and dynamic changes of two anti-inflammatory biomarkers (HLA-DR and IL-10) respectively. $\mathbf{c}$ and $\mathbf{d}$ represented the levels and dynamic changes of two pro-inflammatory biomarkers (IL-6 and IL-8) respectively. * $P<0.05$, ** $P<0.01$, *** $P<0.001$. SI, secondary infection; NSI, non-secondary infection

infection group of this study, HLA-DR expression was lower and IL-10 level showed a trend of increase, which was a sign of immunosuppression. A more severe proinflammatory response in secondary infection group presented as higher levels of IL-6 and IL-8, was also observed in this study. This confirmed the previous conception that higher pro- and anti- inflammatory processes might exist at the same time in septic patients with secondary infection $[21,23]$. Interestingly, we observed a reverse trend of dynamic change between IL-6 and IL-8, though they were both pro-inflammatory cytokines. This might be explained by that the increase of IL- 6 demonstrated the progress of inflammation, as the blood sample collected at day 3 and 7 were more often from severely ill patients. IL-8, as we hypothesized, might be involved in early phase inflammatory process rather than later phase and thus showed a trend of decrease. As the dynamic changes were only statistical significant between certain points in time, studies with larger sample size are necessary to further the study. Those results enlightened us that the identification and risk stratification of immunosuppression and the therapies that boost immunity could be beneficial to the prevention of secondary infection $[13,43,44]$.

We found that secondary infection prolonged the hospitalization time using a multistate model, which could be a result of the complexity of disease requiring longer inhospital treatment and longer LOS in turn increased the risk of secondary infection. Multivariate analysis of our study also revealed that secondary infection was an independent risk factor of in-hospital death. Survival analysis further demonstrated that patients with secondary infection had worse prognosis after first 15 days. In the first 15 days, secondary infection group even had better survival and this could be explained by that patients who were severely sick died earlier before they developed secondary infections. This was consistent with the previous concept that the mortality of patients who survived that early period was more likely affected by secondary infection [13]. A re-increased 

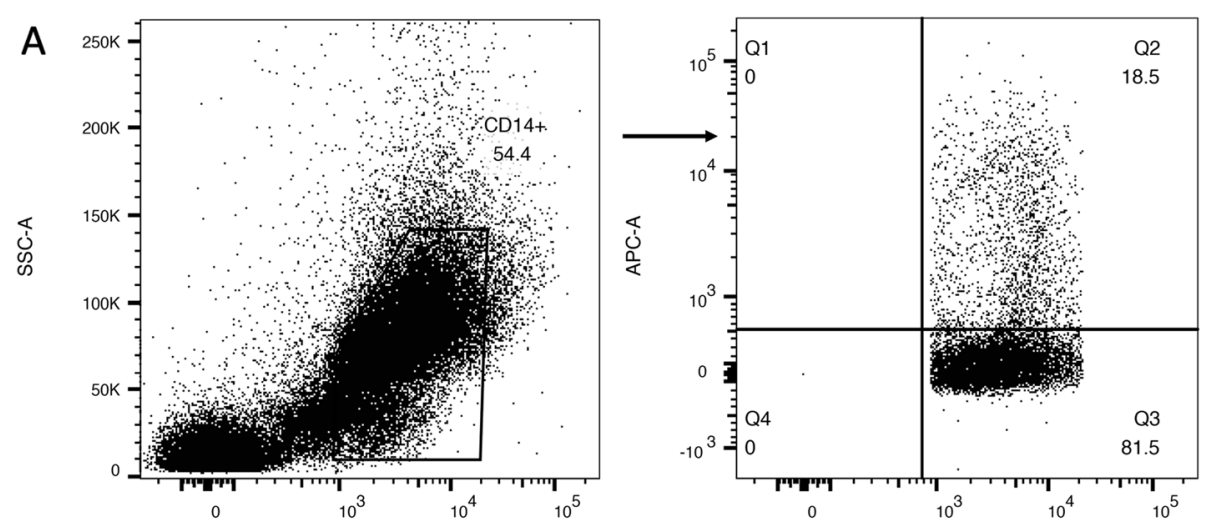

FITC-A
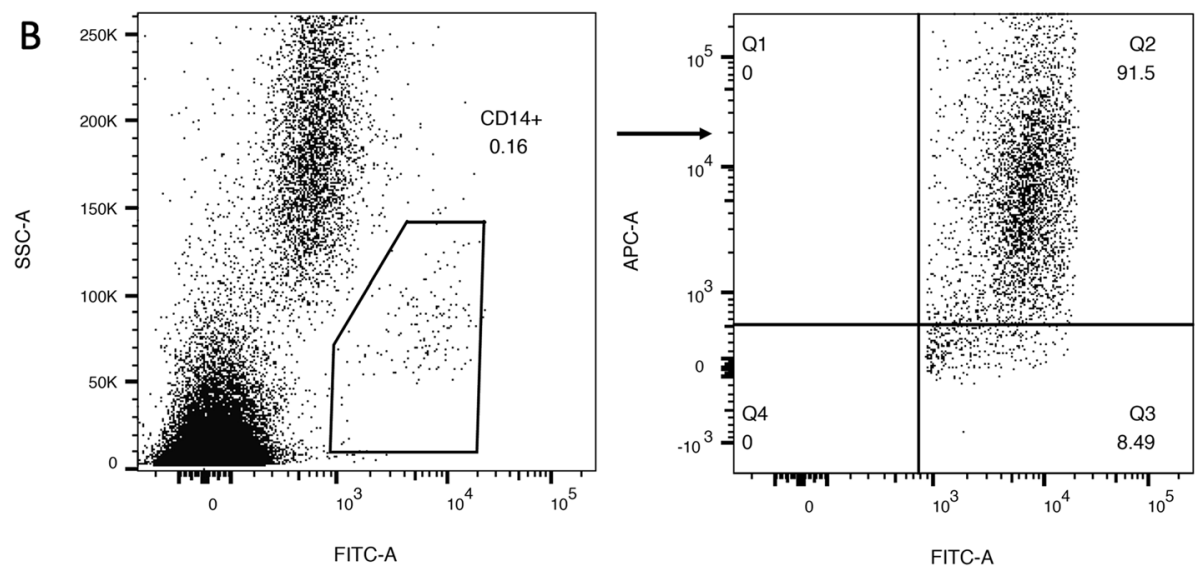

Fig. 3 Representative plots of monocyte HLA-DR measurement by flow cytometry. Monocyte HLA-DR expression was measured by flow cytometry. The samples were collected at day 3 after admission. a The left dot-plot (SSC vs. FITC) delimited the monocytic region. The right dotplot (APC vs. FITC) delimited the CD14 $4^{+}$HLA-DR ${ }^{+}$monocyte (upper right region). The analysis was performed on a patient with immunosuppression as was reflected by the decreased proportion of CD14 $4^{+} \mathrm{HLA}-\mathrm{DR}{ }^{+}$monocyte (18.5\%). $\mathbf{b}$ The same strategy of analysis was used on a patient without immunosuppression. FITC, fluorescein isothiocyanate; APC, allophycocyanin; SSC, side scatter

microbiological burden revealed by positive blood cultures at later phase of sepsis (> 15 days) was observed in the study by Otto et al., which was indicative of secondary infection and poor outcomes [45]. However, Goldenberg et al. addressed that secondary infection was not the main cause of death in sepsis as they found only a small portion (14\%) of septic patients died with an evidence of secondary infection. Some studies found that mitochondrial dysfunction, microvascular leak or even activity of daily living could serve as causes of death from sepsis $[5,28]$.

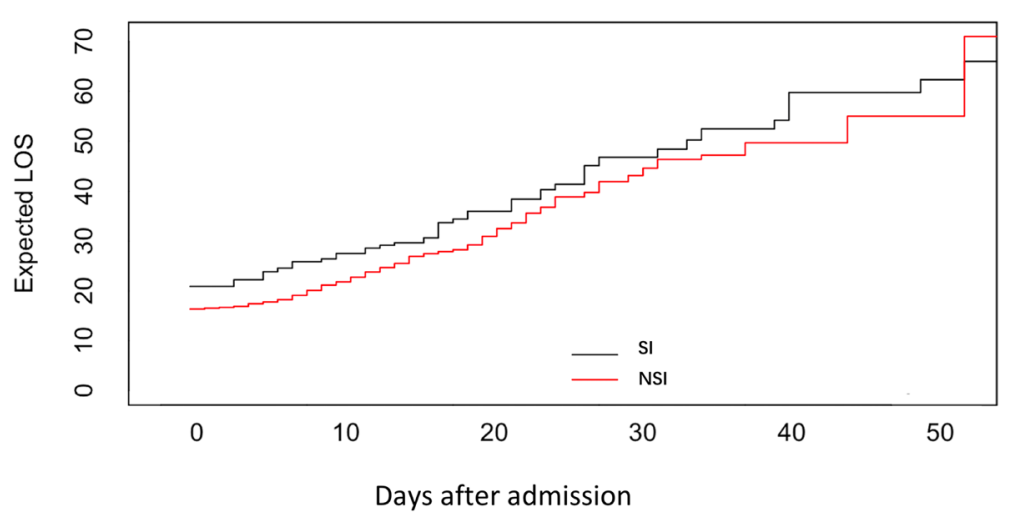

Fig. 4 Expected length of stay of septic patients with and without secondary infection. SI, secondary infection; NSI, non-secondary infection 


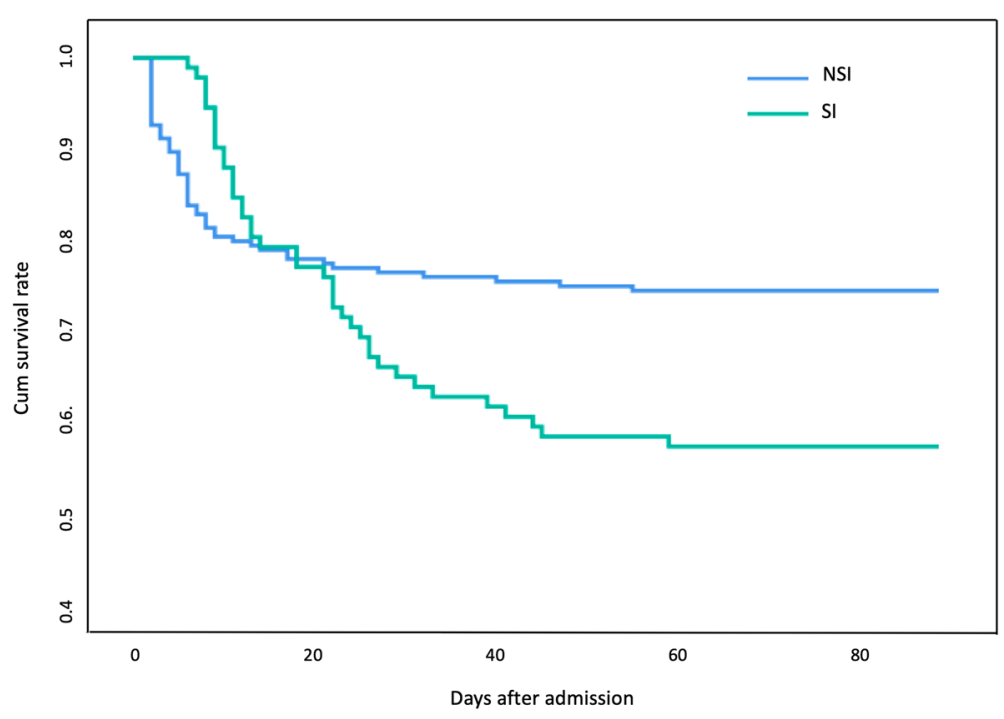

Fig. 5 Kaplan-Meier survival curves of overall septic patients before day 90. SI, secondary infection; NSI, non-secondary infection

This study had some limitations. First, the sample size was relatively small as a single-center study. Second, some clinical data such as the use of antibiotics, the exact dose of glucocorticoids, duration of mechanical ventilation and catheter indwelling were not documented due to the limited medical records, which blocked us from exploring the dose-response relationship. Third, data of HLA-DR expression and serum cytokines levels of many patients were not available as a retrospective study. Thus, the clinical characteristics, risk factors, immune status and prognosis of secondary infection of sepsis were worthy of further prospective research with a larger sample size.

\section{Conclusions}

Invasive operations such as urinary catheterization and deep venous catheterization increased the risk of developing secondary infection, in which underlying immunosuppression also played a role. Secondary infection affected outcomes of patients as it prolonged expected in-hospital LOS and increased mortality in patients who survived early period of sepsis. The monitoring of immune status and proper care to minimize the invasion of pathogens were keys to lower incidence of secondary infection.

\section{Supplementary information}

Supplementary information accompanies this paper at https://doi.org/10. 1186/s12871-019-0849-9.

Additional file 1: Figure S1. Illustration of multistate model to explore the expected length of stay. Patients without secondary infection would move from state 0 to state 2 or state 3 . Patients with secondary infection would move from state 0 to state 1 , and then to state 2 or state 3 .

Additional file 2: Table S1. Time of onset, pathogen and diagnostic criterion of each secondary infection.
Additional file 3: Table S2. Results of the comparison of the change of HLA-DR expression and serum cytokines levels.

Additional file 4: Table S3. Characteristics of the septic patients classified according to the prognosis.

Additional file 5: Table S4. Results of multivariate logistic regression test of risk factors of the in-hospital death.

Additional file 6: Figure S2. Kaplan-Meier survival curves of septic patients after admission. (A) Survival curves of overall septic patients before day 15; (B) Survival curves of septic patients without septic shock before day 15; (C) Survival curves of septic patients with septic shock before day 15; (D) Survival curves of overall septic patients before day 30; (E) Survival curves of septic patients without septic shock before day 30; (F) Survival curves of septic patients with septic shock before day 30; (G) Survival curves of septic patients without septic shock before day 90; (H) Survival curves of septic patients with septic shock before day 90 .

Additional file 7: Figure S3. Kaplan-Meier survival curves of septic patients after day 15. Cumulative survival rate was considered as 1 at day 15. (A) Survival curves of overall septic patients between day 15 and 30; (B) Survival curves of septic patients without septic shock between day 15 and 30; (C) Survival curves of septic patients with septic shock between day 15 and 30; (D) Survival curves of overall septic patients between day 15 and 90; (E) Survival curves of septic patients without septic shock between day 15 and 90; (F) Survival curves of septic patients with septic shock between day 15 and 90 .

\section{Abbreviations}

APACHE II: Acute Physiology and Chronic Health Evaluation II; APC: Allophycocyanin; CDC: Centers for Disease Control and Prevention; Cl: Confidential interval; CKD: Chronic kidney disease; CPOE: Computerized Physician Order Entry; ELISA: Enzyme-linked immunosorbent assay; EMRS: Electronic Medical Record System; FITC: Fluorescein-5-isothiocyanate; HLA-DR: Human leukocyte antigen-D related; ICU: Intensive care unit; IL: Interleukin; LOS: Length of stay; MDSC: Myeloid-derived suppressor cells; MODS: Multiple organ dysfunction syndrome; NHSH: National Healthcare Safety Network; SD: Standard deviation; SOFA: Sequential Organ Failure Assessment; TNF-a: Tumor necrosis factor-a; TRIM: Transfusion-related immune modulation

\section{Acknowledgments}

We would like to thank Prof. Weibing Wang for his help in statistical analysis and Prof. Yiqun Chen for his help in English language editing. 


\section{Authors' contributions}

$Z \mathrm{~S}, \mathrm{CT}, \mathrm{SD}, \mathrm{YC}, \mathrm{YH}$ and JZ contributed to the study design. YC, YH, JZ, YS, JH, $J Y, P W, Y F$ and SL contributed to the data collection, statistical analysis and the interpretation of the results. JH, YY and LY performed the experimental analyses of measurements of monocyte HLA-DR expression and serum cytokines levels. YC, YH, JZ, YS, JW, YY, KL, ZS, CT and SD contributed to the drafting and revision of the manuscript. All authors have approved the final draft of the manuscript.

\section{Funding}

This work was supported by National Natural Science Foundation of China (81471840, 81171837), the Shanghai Traditional Medicine Development Project (ZY3-CCCX3-3018, ZHYY-ZXYJH-201615), Key Project of Shanghai Municipal Health Bureau (2016ZB0202) and the Shanghai Municipal Planning Commission of and Research Fund (20134Y023). The funders had no role in the design of the study nor in the collection, analysis and interpretation of data or in writing the manuscript.

\section{Availability of data and materials}

The datasets used and/or analyzed during the current study are available from the corresponding author on reasonable request.

\section{Ethics approval and consent to participate}

The clinical study protocol was approved by the Ethics Committee Study Board of Zhongshan Hospital, Fudan University, Shanghai, China (record number 2006-23). The Ethics Committee also granted administrative permission to access and use patients' data.

\section{Consent for publication}

Not applicable

\section{Competing interests}

The authors declare that they have no competing interests.

\section{Author details}

'Department of Emergency Medicine, Zhongshan Hospital, Fudan University, Shanghai 200032, China. ${ }^{2}$ Department of Gastroenterology, Zhongshan Hospital, Fudan University, Shanghai 200032, China. ${ }^{3}$ Department of Pharmacology, University of Virginia School of Medicine, Charlottesville, Virginia 22908, USA.

Received: 23 December 2018 Accepted: 13 September 2019 Published online: 18 October 2019

\section{References}

1. Kempker JA, Martin GS. The changing epidemiology and definitions of sepsis. Clin Chest Med. 2016;37(2):165-79.

2. Singer M, Deutschman CS, Seymour CW, Shankar-Hari M, Annane D, Baue $M$, et al. The third international consensus definitions for sepsis and septic shock (Sepsis-3). JAMA. 2016:315(8):801-10.

3. Zhao GJ, Li D, Zhao Q, Song JX, Chen XR, Hong GL, et al. Incidence, risk factors and impact on outcomes of secondary infection in patients with septic shock: an 8-year retrospective study. Sci Rep. 2016;6:38361.

4. van Vught LA, Klein Klouwenberg PM, Spitoni C, Scicluna BP, Wiewel MA, Horn J, et al. Incidence, risk factors, and attributable mortality of secondary infections in the intensive care unit after admission for Sepsis. JAMA. 2016; 315(14):1469-79.

5. Goldenberg NM, Leligdowicz A, Slutsky AS, Friedrich JO, Lee WL. Is nosocomial infection really the major cause of death in sepsis? Crit Care. 2014;18(5):540

6. Daviaud F, Grimaldi D, Dechartres A, Charpentier J, Geri G, Marin N, et al. Timing and causes of death in septic shock. Ann Intensive Care. 2015;5(1):16.

7. Boomer JS, To K, Chang KC, Takasu O, Osborne DF, Walton AH, et al. Immunosuppression in patients who die of sepsis and multiple organ failure. JAMA. 2011;306(23):2594-605.

8. Venet $F$, Monneret $G$. Advances in the understanding and treatment of sepsis-induced immunosuppression. Nat Rev Nephrol. 2017;14(2):121-37.

9. Leentjens J, Kox M, van der Hoeven JG, Netea MG, Pickkers P. Immunotherapy for the adjunctive treatment of sepsis: from immunosuppression to immunostimulation. Time for a paradigm change? Am J Respir Crit Care Med. 2013;187(12):1287-93.
10. Gurung P, Rai D, Condotta SA, Babcock JC, Badovinac VP, Griffith TS Immune unresponsiveness to secondary heterologous bacteria infection after sepsis induction is TRAIL dependent. J Immunol. 2011; 187(5):2148-54.

11. Hotchkiss RS, Monneret G, Payen D. Sepsis-induced immunosuppression: from cellular dysfunctions to immunotherapy. Nat Rev Immunol. 2013; 13(12):862-74

12. Xiu B, Lin Y, Grote DM, Ziesmer SC, Gustafson MP, Maas ML, et al. IL10 induces the development of immunosuppressive CD14(+)HLADR(low/-) monocytes in B-cell non-Hodgkin lymphoma. Blood Cancer 2015:5:e328.

13. Venet F, Lukaszewicz AC, Payen D, Hotchkiss R, Monneret G. Monitoring the immune response in sepsis: a rational approach to administration of immunoadjuvant therapies. Curr Opin Immunol. 2013;25(4):477-83.

14. Zhuang YG, Peng $H$, Chen $Y Z$, Zhou SQ, Chen YQ. Dynamic monitoring of monocyte HLA-DR expression for the diagnosis, prognosis, and prediction of sepsis. Front Biosci (Landmark Ed). 2017:22:1344-54.

15. Lukaszewicz AC, Grienay M, Resche-Rigon M, Pirracchio R, Faivre V, Boval B, et al. Monocytic HLA-DR expression in intensive care patients: interest for prognosis and secondary infection prediction. Crit Care Med. 2009:37(10):2746-52.

16. Chenouard A, Braudeau C, Cottron N, Bourgoin P, Salabert N, Roquilly A, et al. HLA-DR expression in neonates after cardiac surgery under cardiopulmonary bypass: a pilot study. Intensive Care Med Exp. 2018;6(1):1.

17. Schefold JC. Measurement of monocytic HLA-DR (mHLA-DR) expression in patients with severe sepsis and septic shock: assessment of immune organ failure. Intensive Care Med. 2010;36(11):1810-2

18. Landelle C, Lepape A, Voirin N, Tognet E, Venet F, Bohe J, et al. Low monocyte human leukocyte antigen-DR is independently associated with nosocomial infections after septic shock. Intensive Care Med. 2010; 36(11):1859-66

19. Drewry AM, Ablordeppey EA, Murray ET, Beiter ER, Walton AH, Hall MW et al. Comparison of monocyte human leukocyte antigen-DR expression and stimulated tumor necrosis factor alpha production as outcome predictors in severe sepsis: a prospective observational study. Crit Care. 2016;20(1):334.

20. Ohno Y, Kitamura H, Takahashi N, Ohtake J, Kaneumi S, Sumida K, et al. IL-6 down-regulates HLA class II expression and IL-12 production of human dendritic cells to impair activation of antigen-specific CD4(+) T cells. Cancer Immunol Immunother. 2016;65(2):193-204.

21. Lekkou A, Karakantza M, Mouzaki A, Kalfarentzos F, Gogos CA. Cytokine production and monocyte HLA-DR expression as predictors of outcome for patients with community-acquired severe infections. Clin Diagn Lab Immunol. 2004;11(1):161-7.

22. Monneret $G$, Finck M-E, Venet F, Debard AL, Bohé J, Bienvenu J, et al. The anti-inflammatory response dominates after septic shock: association of low monocyte HLA-DR expression and high interleukin-10 concentration. Immunol Lett. 2004;95(2):193-8.

23. van Vught LA, Wiewel MA, Hoogendijk AJ, Frencken JF, Scicluna BP, Klein Klouwenberg PMC, et al. The host response in patients with sepsis developing intensive care unit-acquired secondary infections. Am J Respir Crit Care Med. 2017;196(4):458-70.

24. Mera S, Tatulescu D, Cismaru C, Bondor C, Slavcovici A, Zanc V, et al. Multiplex cytokine profiling in patients with sepsis. APMIS. 2011;119(2):155-63.

25. Horan TC, Andrus M, Dudeck MA. CDC/NHSN surveillance definition of health care-associated infection and criteria for specific types of infections in the acute care setting. Am J Infect Control. 2008;36(5):309-32.

26. Allignol A. Package 'etm'. 2018. https://cran.r-project.org/web/packages/ etm/etm.pdf. Accessed 19 July 2018.

27. Wang J, Liu F, Tartari E, Huang J, Harbarth S, Pittet D, et al. The prevalence of healthcare-associated infections in mainland China: a systematic review and meta-analysis. Infect Control Hosp Epidemiol. 2018;39(6):701-9.

28. Allegranzi B, Nejad SB, Combescure C, Graafmans W, Attar H, Donaldson L, et al. Burden of endemic health-care-associated infection in developing countries: systematic review and meta-analysis. Lancet. 2011;377(9761):228-41.

29. Jeong SJ, Yoon SS, Han SH, Yong DE, Kim CO, Kim JM. Evaluation of humoral immune response to nosocomial pathogen and functional status in elderly patients with sepsis. Arch Gerontol Geriatr. 2014:58(1):10-4

30. Delano MJ, Thayer T, Gabrilovich S, Kelly-Scumpia KM, Winfield RD, Scumpia $\mathrm{PO}$, et al. Sepsis induces early alterations in innate immunity that impact mortality to secondary infection. J Immunol. 2010;186(1):195-202. 
31. Koch RM, Kox M, de Jonge Ml, van der Hoeven JG, Ferwerda G, Pickkers P. Patterns in bacterial- and viral-induced immunosuppression and secondary infections in the ICU. Shock. 2017:47:5-12.

32. Wolkewitz M, Cooper BS, Palomar-Martinez M, Alvarez-Lerma F, OlaecheaAstigarraga P, Barnett AG, et al. Multilevel competing risk models to evaluate the risk of nosocomial infection. Crit Care. 2014;18(2):R64.

33. Tao L, Hu B, Rosenthal VD, Gao X, He L. Device-associated infection rates in 398 intensive care units in Shanghai, China: international nosocomial infection control consortium (INICC) findings. Int J Infect Dis. 2011;15(11):e774-80.

34. Stevens V, Geiger K, Concannon C, Nelson RE, Brown J, Dumyati G. Inpatient costs, mortality and 30-day re-admission in patients with central-lineassociated bloodstream infections. Clin Microbiol Infect. 2014;20(5):0318-24.

35. Olaechea PM, Palomar M, Alvarez-Lerma F, Otal JJ, Insausti J, Lopez-Pueyo $\mathrm{MJ}$, et al. Morbidity and mortality associated with primary and catheterrelated bloodstream infections in critically ill patients. Rev Esp Quimioter. 2013;26(1):21-9

36. Temiz E, Piskin N, Aydemir H, Oztoprak N, Akduman D, Celebi G, et al. Factors associated with catheter-associated urinary tract infections and the effects of other concomitant nosocomial infections in intensive care units. Scand J Infect Dis. 2012;44(5):344-9.

37. Mehta A, Bhagat R. Preventing ventilator-associated infections. Clin Chest Med. 2016;37(4):683-92.

38. Dupuis C, Sonneville R, Adrie C, Gros A, Darmon M, Bouadma L, et al. Impact of transfusion on patients with sepsis admitted in intensive care unit: a systematic review and meta-analysis. Ann Intensive Care. 2017;7(1):5.

39. Cervia JS, Wenz B, Ortolano GA. Leukocyte reduction's role in the attenuation of infection risks among transfusion recipients. Clin Infect Dis. 2007;45(8):1008-13.

40. Cata JP, Wang H, Gottumukkala V, Reuben J, Sessler DI. Inflammatory response, immunosuppression, and cancer recurrence after perioperative blood transfusions. Br J Anaesth. 2013;110(5):690-701.

41. Rice TC, Pugh AM, Caldwell CC, Schneider BSP. Balance between the proinflammatory and anti-inflammatory immune responses with blood transfusion in sepsis. Crit Care Nurs Clin North Am. 2017:29(3):331-40.

42. Bah I, Kumbhare A, Nguyen L, McCall CE, El Gazzar M. IL-10 induces an immune repressor pathway in sepsis by promoting S100A9 nuclear localization and MDSC development. Cell Immunol. 2018;332:32-8.

43. Monneret G, Lepape A, Venet F. Reversing ICU-acquired immunosuppression: an innovative biomarker-guided therapeutic strategy for decreasing sepsis mortality and nosocomial infection rate. Pathol Biol (Paris). 2011;59(6):329-33.

44. Conway Morris A, Datta D, Shankar-Hari M, et al. Cell-surface signatures of immune dysfunction risk-stratify critically ill patients: INFECT study. Intensive Care Med. 2018;44(5):627-35.

45. Otto GP, Sossdorf M, Claus RA, Rodel J, Menge K, Reinhart K, et al. The late phase of sepsis is characterized by an increased microbiological burden and death rate. Crit Care. 2011;15(4):R183.

\section{Publisher's Note}

Springer Nature remains neutral with regard to jurisdictional claims in published maps and institutional affiliations.

Ready to submit your research? Choose BMC and benefit from:

- fast, convenient online submission

- thorough peer review by experienced researchers in your field

- rapid publication on acceptance

- support for research data, including large and complex data types

- gold Open Access which fosters wider collaboration and increased citations

- maximum visibility for your research: over $100 \mathrm{M}$ website views per year

At $\mathrm{BMC}$, research is always in progress.

Learn more biomedcentral.com/submissions 